\title{
Preparation And Characterization Of Core-Shell Cobalt Silver Nanoparticles
}

\section{Michael Giersig}

Hahn-Meitner-Institut Berlin, Department Solar Energy Research;

Glienicker Straße 100, 14109 Berlin, Germany, e-mail :giersig@hmi.de

\begin{abstract}
Monodisperse bimetallic Ag/Co-composite nanocrystals have been prepared using methods of colloid chemistry. Transmission Electron Microscopy (TEM) showed well isolated Ag/Co-particles with a narrow size distribution centered around $10 \mathrm{~nm}$ in diameter. The composition of the particles was determined with Energy Disperse X-Ray Spectrometry (EDX). The typical parameters of both metallic components fcc-structures could be verified within the individual particles using Selected Area

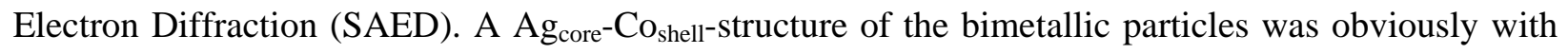
TEM and Electron Energy-Loss Spectroscopy (EELS). $\mathrm{Ag}_{10} \mathrm{Co}_{4.5}$-crystals were found to be ferromagnetic below a blocking temperature of $90 \mathrm{~K}$ and superparamagnetic at room temperature.

\section{Experimental}

AgCo nanoparticles were prepared by thermal decomposition of dicobalt octacarbonyl $\left(\mathrm{Co}_{2}(\mathrm{CO})_{8}\right)$ in the presence of water free silver perchlorate. Octanoic acid and oleic acid were used as stabilizing ligands. The particles are superparamagnetic above the blocking temperature of $90 \mathrm{~K}$ (3). The dispersion phases of the prepared sols were characterized by optical spectroscopy and transmission electron microscopy Philips CM12 equipped witch the slow scan camera and EDAX-analyzer as well as electron energy loss spectroscopy (EELS) Gatan on Philips CM20 field emission gun microscope.
\end{abstract}

\section{Results}

The typical image of the bimetallic particles is shown in Fig.1, in which the relatively monodisperse particles are presented. By the HRTEM we have detected the lattice plane from fcc cobalt and fcc Ag structures. The different ratio of produced particles was first detected by EDAX analysis. The unequivocal determination the core-shell structures was done by the EELS measurement. 


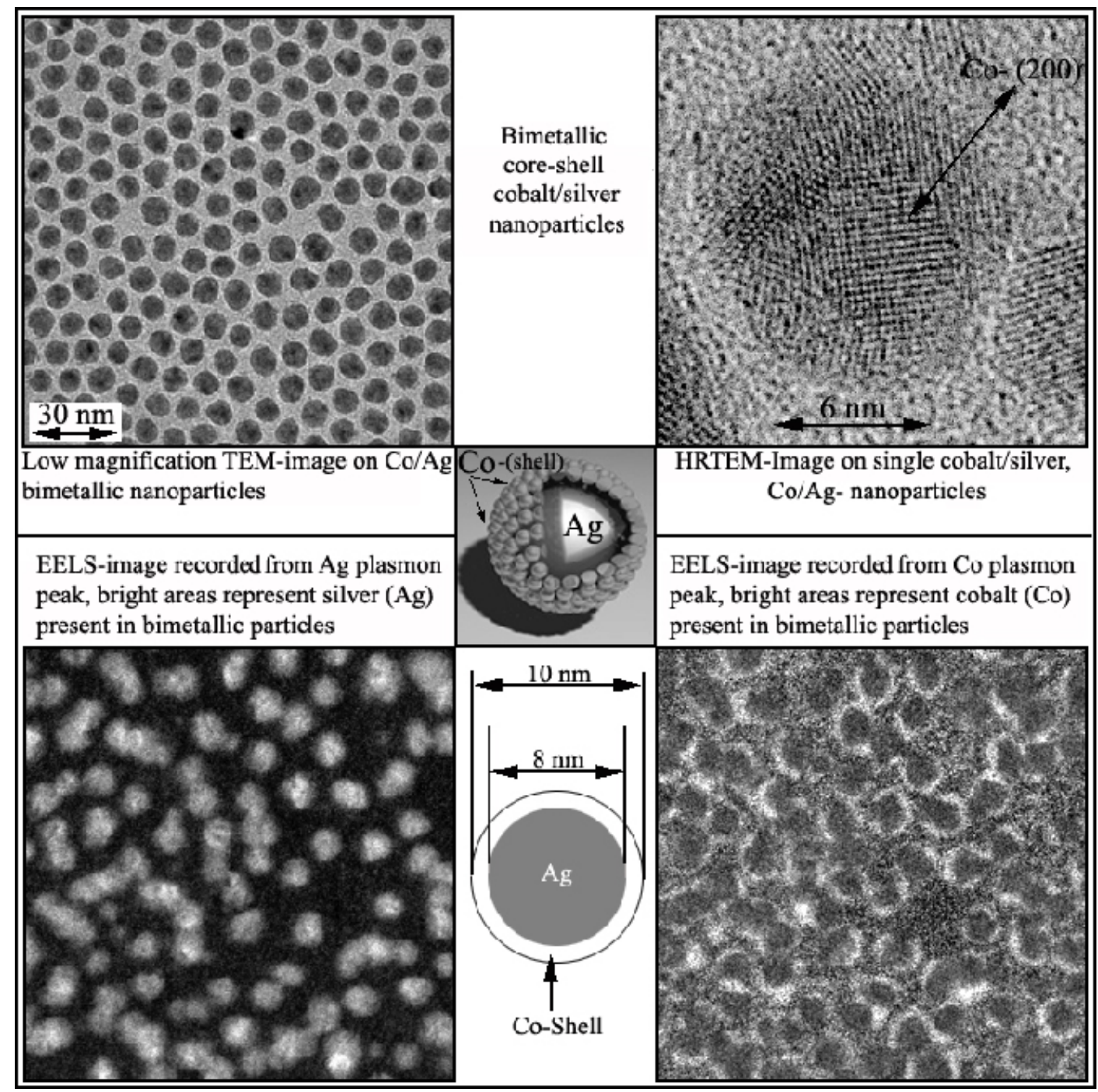

Fig.1. Gallery of pictures from the TEM and EELS analysis on bimetallic Co/Ag nanoparticles

\section{References:}

1. Giersig, M, and Hilgendorff, M., (1999) J. Phys.D:Appl.Phys.32 L111

2. Giersig, M., and Hilgendorff, M., (2002) Aust.J. Chem.54, 2001, 497-501

3. N. Sobal et all, (2002)submitted for publication to Nano-Letters 